\title{
Adipose Tissue Remodeling as Homeostatic Inflammation
}

\author{
Michiko Itoh, ${ }^{1}$ Takayoshi Suganami, ${ }^{1}$ Rumi Hachiya, ${ }^{1}$ and Yoshihiro Ogawa ${ }^{1,2}$ \\ ${ }^{1}$ Department of Molecular Medicine and Metabolism, Medical Research Institute, Tokyo Medical and Dental University, \\ 1-5-45 Yushima, Bunkyo-ku, Tokyo 113-8510, Japan \\ ${ }^{2}$ Global Center of Excellence Program, International Research Center for Molecular Science in Tooth and Bone Diseases, \\ Tokyo Medical and Dental University, Tokyo 113-8510, Japan
}

Correspondence should be addressed to Yoshihiro Ogawa, ogawa.mmm@mri.tmd.ac.jp

Received 23 March 2011; Accepted 27 April 2011

Academic Editor: Ichiro Manabe

Copyright (c) 2011 Michiko Itoh et al. This is an open access article distributed under the Creative Commons Attribution License, which permits unrestricted use, distribution, and reproduction in any medium, provided the original work is properly cited.

\begin{abstract}
Evidence has accumulated indicating that obesity is associated with a state of chronic, low-grade inflammation. Obese adipose tissue is characterized by dynamic changes in cellular composition and function, which may be referred to as "adipose tissue remodeling". Among stromal cells in the adipose tissue, infiltrated macrophages play an important role in adipose tissue inflammation and systemic insulin resistance. We have demonstrated that a paracrine loop involving saturated fatty acids and tumor necrosis factor- $\alpha$ derived from adipocytes and macrophages, respectively, aggravates obesity-induced adipose tissue inflammation. Notably, saturated fatty acids, which are released from hypertrophied adipocytes via the macrophage-induced lipolysis, serve as a naturally occurring ligand for Toll-like receptor 4 complex, thereby activating macrophages. Such a sustained interaction between endogenous ligands derived from parenchymal cells and pathogen sensors expressed in stromal immune cells should lead to chronic inflammatory responses ranging from the basal homeostatic state to diseased tissue remodeling, which may be referred to as "homeostatic inflammation". We, therefore, postulate that adipose tissue remodeling may represent a prototypic example of homeostatic inflammation. Understanding the molecular mechanism underlying homeostatic inflammation may lead to the identification of novel therapeutic strategies to prevent or treat obesity-related complications.
\end{abstract}

\section{Introduction}

The metabolic syndrome is a constellation of visceral fat obesity, insulin resistance, atherogenic dyslipidemia, and hypertension, which all independently increase the risk of atherosclerotic diseases [1-5]. The adipose tissue secretes a number of bioactive substances or adipocytokines, and unbalanced production of pro- and anti-inflammatory adipocytokines in obese adipose tissue may critically contribute to many aspects of the metabolic syndrome [1-5]. Obesity is now viewed as a state of systemic, chronic low-grade inflammation [1-4]. In contrast to acute inflammation which resolves by an active termination program, chronic inflammation may involve persistent stress and/or impaired resolution process, thereby resulting in functional maladaptation and tissue remodeling [6]. On the other hand, during the course of obesity, adipose tissue is characterized by adipocyte hypertrophy, followed by increased angiogenesis, immune cell infiltration, and extracellular matrix overproduction
$[1,2,7,8]$, which may be referred to as adipose tissue remodeling.

Pathogen sensors or pattern-recognition receptors (PRRs), which are important for the recognition of pathogen-associated molecular patterns (PAMPs) in innate immunity, are also capable of recognizing endogenous ligands, damage-associated molecular patterns (DAMPs) or danger signals (Figure 1) $[6,9,10]$. Interaction between endogenous ligands and pathogen sensors may play a role in the basal homeostatic state as well as diseased tissue remodeling, which has been referred to as homeostatic inflammation $[6,11]$. This paper summarizes the molecular mechanism and pathophysiologic implication of adipose tissue remodeling as a prototypic example of homeostatic inflammation.

\section{Adipose Tissue Inflammation and Adipose Tissue Remodeling}

In addition to lipid-laden mature adipocytes, the adipose tissue is composed of various stromal cells, including 


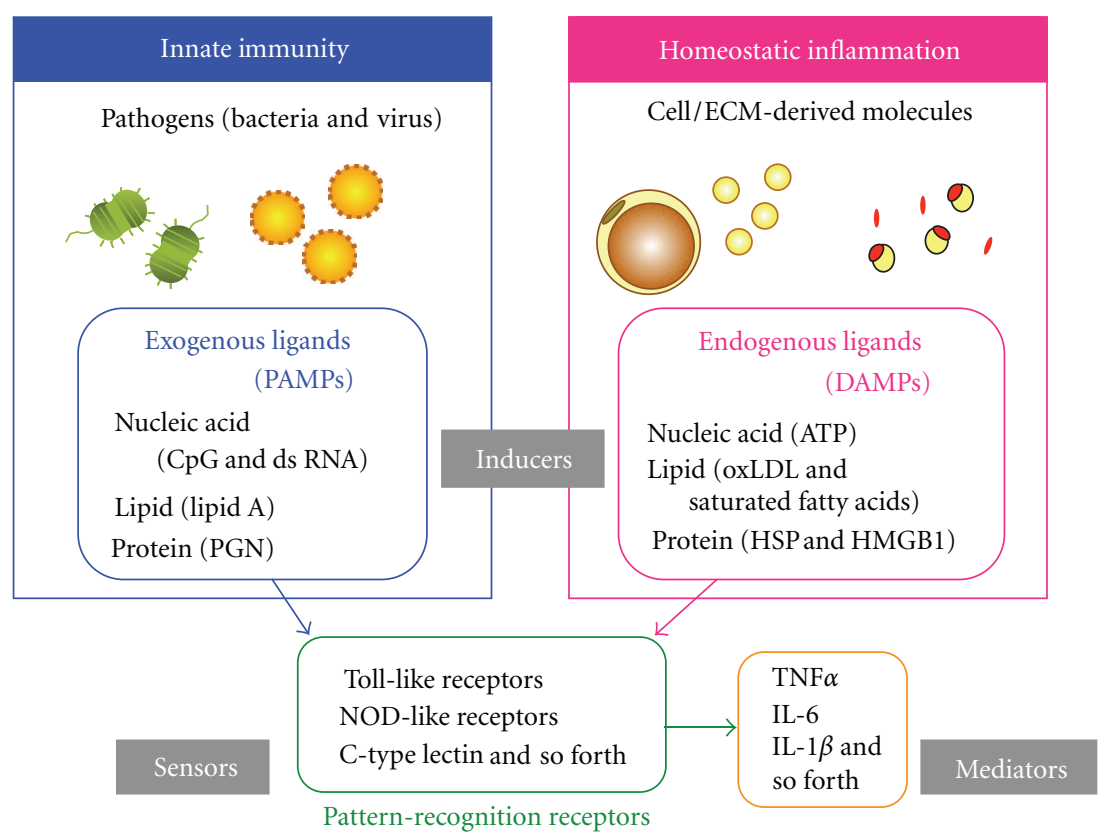

FIGURE 1: Adipose tissue inflammation as homeostatic inflammation. In innate immunity, exogenous ligands (pathogen-associated molecular patterns; PAMPs) are sensed by pattern-recognition receptors (PRRs), thereby inducing inflammatory changes. On the other hand, damage-associated molecular patterns (DAMPs) released from damaged or stressed cells and tissues can activate PRRs, thereby inducing homeostatic inflammation ranging from the basal homeostatic state to diseased tissue remodeling. For instance, free fatty acids (FFAs) released from hypertrophied adipocytes can report, as a danger signal, their diseased state to macrophages via Toll-like receptor 4 (TLR4) complex during the course of obesity. dsRNA, double-strand RNA; PGN, peptidoglycan; ATP, adenosine tri-phosphate; oxLDL, oxidized low-density lipoprotein; HSP, heat shock protein; HMGB1, high-mobility group box-1.

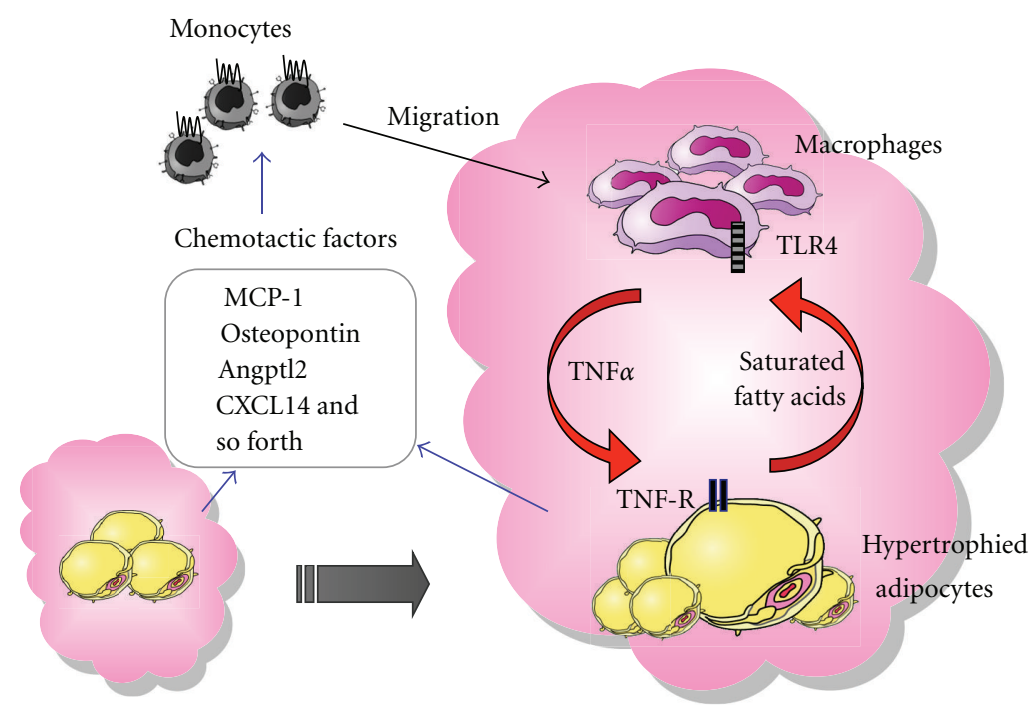

FIGURE 2: Molecular mechanism underlying adipose tissue inflammation. During the course of obesity, adipose tissue secretes several chemotactic factors to induce macrophage infiltration into adipose tissue. Circulating monocytes migrate and infiltrate into adipose tissue through adhesion process to endothelial cells. Macrophages enhance the inflammatory changes through the crosstalk with parenchymal adipocytes. For example, the macrophage-derived tumor necrosis factor- $\alpha$ (TNF $\alpha$ ) induces the release of saturated fatty acids from adipocytes via lipolysis, which, in turn, induces inflammatory changes in macrophages via TLR4. Such a paracrine loop between adipocytes and macrophages constitutes a vicious cycle, thereby further accelerating adipose tissue inflammation. TNF-R, TNF $\alpha$ receptor. 
preadipocytes, endothelial cells, fibroblasts, and immune cells [12]. Obese adipose tissue exhibits functional and morphological changes, thereby leading to unbalanced production of pro- and anti-inflammatory adipocytokines $[1,2,7$, 8]. The morphological changes found in obese adipose tissue are reminiscent of the chronic inflammatory responses in atherosclerotic vascular walls termed vascular remodeling, which arise from the complex interactions among vascular endothelial cells, vascular smooth muscle cells, lymphocytes, and monocyte-derived macrophages [4]. Vascular remodeling is considered to be an adaptive process in response to long-term changes in hemodynamic conditions and lipid metabolism, thereby contributing to the pathophysiology of vascular diseases [13]. Thus, the dynamic changes seen in obese adipose tissue can be referred to as adipose tissue remodeling. Notably, macrophage infiltration and inflammation-related gene expression in the adipose tissue precedes the development of insulin resistance in animal models [14, 15], suggesting that macrophages should play a central role in adipose tissue remodeling. It is, therefore, important to know the pathophysiologic role of macrophages infiltrated into the adipose tissue during the course of adipose tissue remodeling.

\section{Macrophage Infiltration into Obese Adipose Tissue}

Evidence has accumulated that adipocytes per se secrete proinflammatory cytokines and chemokines, such as tumor necrosis factor- $\alpha$ (TNF $\alpha$ ), interleukin-6 (IL-6), and monocyte chemoattractant protein-1 (MCP-1), during the course of adipocyte hypertrophy [1-3]. Increased production of chemokines in obese adipose tissue has been implicated in the regulation of monocyte recruitment to adipose tissue [14]. The involvement of MCP-1/chemokine receptor 2 (CCR2) pathway has been extensively studied as the mechanism underlying macrophage infiltration into obese adipose tissue (Figure 2) [16-19]. Moreover, several reports have suggested the role of other chemotactic factors in obesity-induced macrophage infiltration: osteopontin, angiopoietin-like protein 2 (Angptl2), and CXC motif chemokine ligand-14 (CXCL14) (Figure 2) [20-22]. Inhibition of macrophage infiltration into obese adipose tissue through genetic and/or pharmacologic strategies has improved the dysregulation of adipocytokine production, thereby leading to the amelioration of obesity-induced adipose tissue inflammation and insulin resistance. Indeed, macrophage infiltration and inflammationrelated gene expression in the adipose tissue precedes the development of insulin resistance in animal models $[14,15]$. Understanding the molecular mechanisms underlying increased macrophage infiltration into obese adipose tissue may lead to the identification of novel therapeutic strategies to prevent or treat obesity-induced adipose tissue inflammation.

\section{Interaction between Adipocytes and Macrophages}

The adipose tissue macrophages also represent a major source of pro-inflammatory cytokines, which play important roles in chronic inflammatory responses in obese adipose tissue. Using an in vitro coculture system composed of adipocytes and macrophages, we have demonstrated that a paracrine loop involving saturated fatty acids and TNF $\alpha$ derived from adipocytes and macrophages, respectively, establishes a vicious cycle that augments the inflammatory changes (Figure 2) [23]. Among numerous cytokines derived from infiltrated macrophages in obese adipose tissue, TNF $\alpha$ acts on TNF receptor in hypertrophied adipocytes, thereby inducing pro-inflammatory cytokine production and adipocyte lipolysis via nuclear factor- $\kappa \mathrm{B}-(\mathrm{NF}-\kappa \mathrm{B}-)$ dependent and independent (possibly mitogen-activated protein kinase- (MAPK-) dependent) mechanisms, respectively [24]. On the other hand, saturated fatty acids released from adipocytes serve as a naturally occurring ligand for Tolllike receptor 4 (TLR4) complex, which is essential for the recognition of lipopolysaccharide (LPS), to induce NF- $\kappa \mathrm{B}$ activation in macrophages [24].

The interaction between adipocytes and macrophages results in marked upregulation of pro-inflammatory adipocytokines and significant downregulation of anti-inflammatory adipocytokines, which lead to development of obesityrelated complications in multiple organs, such as atherosclerosis and hepatic steatosis [1-4]. For instance, adiponectin is a well-established anti-inflammatory adipocytokine, which is markedly downregulated in obese adipose tissue, and supplementation of adiponectin in obese mice effectively reverses insulin resistance in the skeletal muscle and liver [25, 26]. On the other hand, overproduction of MCP-1 induces macrophage infiltration into the adipose tissue and directly induces insulin resistance in the skeletal muscle and liver [17, $18,27]$. Thus, dysregulation of adipocytokine production as a result of inflammatory changes in the adipose tissue may be involved in the pathogenesis of metabolic derangements in obesity.

\section{Heterogeneity of Adipose Tissue Macrophages}

Recent studies have pointed to the phenotypic change of macrophages in lean and obese adipose tissue; M1 or classically activated (pro-inflammatory) macrophages and M2 or alternatively activated (anti-inflammatory) macrophages (Figure 3) [28]. Adipocytes in lean adipose tissue produce humoral factors that induce M2 activation of macrophages, such as interleukin-4 (IL-4) and interleukin-13 (IL-13), and M2 activated macrophages release anti-inflammatory mediators, such as interleukin (IL-10) [29]. On the other hand, hypertrophied adipocytes secrete pro-inflammatory saturated fatty acids, cytokines, and chemokines to induce M1 polarization of macrophages [29]. Activated M1 macrophages in turn produce pro-inflammatory cytokines and 


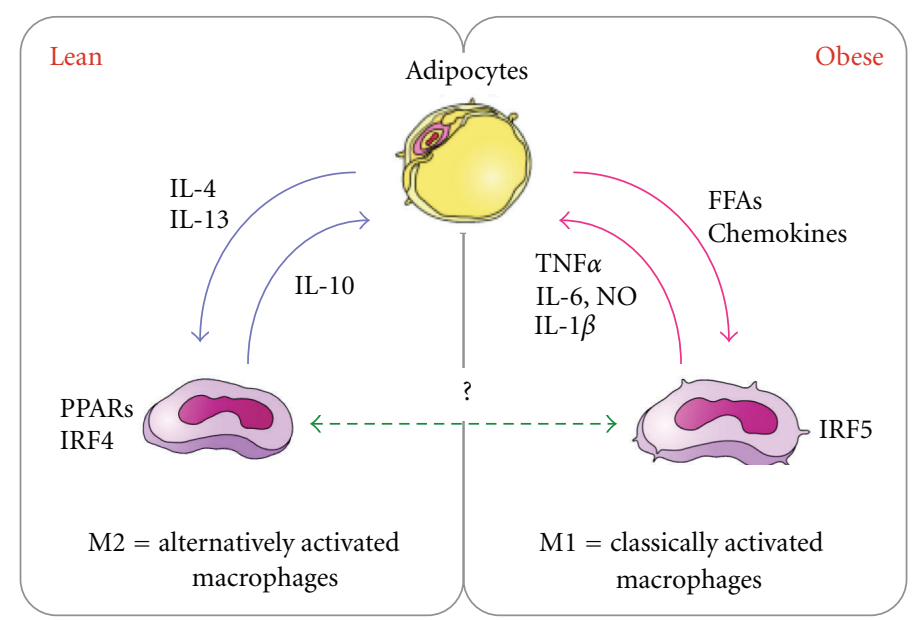

FIGURE 3: Regulation of macrophage polarity in adipose tissue. Recent evidence has also pointed to the heterogeneity of adipose tissue macrophages, that is, M1 or classically activated (pro-inflammatory) macrophages and M2 or alternatively activated (anti-inflammatory) macrophages. Under lean condition, adipocytes secrete factors that promote M2 activation of macrophages, such as interleukin-4 (IL) and interleukin-13 (IL-13). M2 macrophages secrete anti-inflammatory mediators. On the other hand, adipocytes secrete pro-inflammatory FFAs, chemokines, and cytokines under obese condition. Activated M1 macrophages produce large amounts of pro-inflammatory cytokines, thereby accelerating inflammatory responses in adipose tissue through paracrine interaction between adipocytes and macrophages.

chemokines, thereby accelerating adipose tissue inflammation.

We have recently identified activating transcription factor 3 (ATF3), a member of ATF/cAMP response element-binding protein family of basic leucine zipper-type transcription factors, as a target gene of saturated fatty acids/TLR4 signaling in adipose tissue macrophages and found that ATF3 attenuates obesity-induced macrophage activation in obese adipose tissue [30]. On the other hand, peroxisome proliferator-activated receptor $\gamma(\operatorname{PPAR} \gamma)$ and peroxisome proliferator-activated receptor $\beta / \delta(\operatorname{PPAR} \beta / \delta)$ can stimulate $\mathrm{M} 2$ polarization of adipose tissue macrophages and thus systemic insulin sensitivity [31-34]. Indeed, the activation of PPAR $\gamma$ by pioglitazone, a thiazolidinedione class of insulin sensitizer, improves the unbalanced M1/M2 phenotype of adipose tissue macrophages in diet-induced obese mice [35]. Interestingly, circulating blood monocytes, precursors of infiltrated macrophages to the site of chronic inflammation, also express both M1 and M2 markers [36, 37]. Moreover, monocytes in obese mice and/or obese type 2 diabetic patients show significantly higher expression of M1 markers and lower expression of M2 markers relative to normalweight controls [36]. Thus, pioglitazone treatment improves the unbalanced M1/M2 phenotype of monocytes, which may contribute to its antidiabetic and antiatherogenic effect [36, 37].

Recent studies have found other molecules that regulate macrophage polarization; that is, Jumonji domain containing-3 (Jmjd3) is essential for M2 activation through demethylation of interferon-regulatory factor 4 (IRF4) under infectious condition [38], and interferon-regulatory factor 5 (IRF5) is crucial for conversion from M2 to M1 activation in response to LPS [39]. It is interesting to know their importance in the regulation of macrophage polarization and plasticity during the course of obesity. Modulating macrophage activation state in obese adipose tissue would be a novel therapeutic target to treat or prevent the progression of obesityinduced complications such as diabetes and atherosclerosis.

\section{Adipose Tissue Remodeling and Ectopic Lipid Accumulation}

The adipose tissue is primarily an energy reservoir that stores fatty acids in the form of triglyceride, which is facilitated by insulin. However, obesity induces insulin-resistant state and inflammation in the adipose tissue, both of which lead to increased fatty acid release from the adipose tissue [1, 23, 29]. Moreover, recent studies have suggested that increased expression of genes related to ECM components and fibrotic changes in the adipose tissue from obese subjects and animals [40-43]. It is reported that adipose tissue fibrosis is negatively correlated with adipocyte diameters in human adipose tissue [44], suggesting that increased ECM components may limit adipose tissue expandability. Indeed, Khan et al. reported that mice lacking collagen VI, which is expressed predominantly in the adipose tissue, exhibit the uninhibited adipose tissue expansion and substantial improvements in whole-body energy homeostasis during a high-fat diet feeding [43]. It is conceivable that the rigid extracellular environment limits adipocyte expansion, and triggers adipocyte cell death and inflammatory responses through MAPK activation by increased shear stress and membrane stretching $[43,44]$. Recent evidence suggests that impaired lipid storage in the adipose tissue may contribute to ectopic lipid accumulation in the skeletal muscle, liver, and pancreatic $\beta$-cells, where lipotoxicity impairs their metabolic functions [45-47]. This discussion supports the emerging view that metabolic problems associated with obesity become overt when adipose tissue cannot fully meet demands for 
additional lipid storage in addition to the dysregulation of adipocytokine production.

\section{Adipose Tissue Remodeling as Homeostatic Inflammation}

TLR4 is a pattern-recognition receptor essential for the recognition of LPS, which is reported to play an important role in obesity-induced adipose tissue inflammation and systemic glucose and lipid metabolism in vivo [24, 48-50]. In obese adipose tissue, TLR4 expressed in macrophages is capable of sensing saturated fatty acids (FAs) released from adipocytes to induce chronic inflammatory responses $[24,51,52]$, suggesting that saturated fatty acids could be a danger signal. On the other hand, free fatty acids (FFAs) are an important energy source mobilized from triglycerides stored in the adipose tissue, particularly under starvation conditions. Kosteli et al. have recently suggested that FFAs released from adipocytes during fasting recruit macrophages into the adipose tissue, which may be involved in the regulation of local lipid concentrations [53]. In this regard, FFAs, when released physiologically during fasting or starvation via adipocyte lipolysis, may be involved in the regulation of metabolic homeostasis within the adipose tissue rather than a danger signal. Under overnutrition conditions, increased concentrations of FFAs also activate inflammatory pathways to maintain adipose tissue homeostasis such as tissue repair and regulation of metabolism. When cellular and/or tissue stresses are excessive and/or sustained and adaptive responses are no longer possible, inflammatory responses are prolonged (i.e., chronic inflammation), thereby leading to diseased tissue remodeling [6].

Recently, we have reported that macrophage-inducible C-type lectin (Mincle; also called Clec4e and Clecsf9), a pathogen sensor for pathogenic fungi and Mycobacterium tuberculosis, is induced in adipose tissue macrophages in obesity at least partly through the saturated fatty acid/TLR4/ NF- $\kappa \mathrm{B}$ pathway, thereby suggesting its pathophysiologic role in obesity-induced adipose tissue inflammation [54]. Yamasaki et al. reported that Mincle serves as a receptor for SAP130, a component of small nuclear ribonucleoprotein released from damaged cells, to sense cell death and induce pro-inflammatory cytokine production [55]. Since dead adipocytes are surrounded by macrophages in the adipose tissue of obese humans and mice (crown-like structure) [7, 8, 56], it is conceivable that Mincle plays a role in sensing adipocytederived endogenous ligand(s) during adipocyte death.

The above discussion supports the concept that interaction between endogenous ligands and pathogen sensors in the adipose tissue involves multiple stages of adipose tissue remodeling, ranging from normal metabolic homeostasis to diseased tissue remodeling, which may be referred to as homeostatic inflammation. It is interesting to identify other endogenous danger signals and pathogen sensors that contribute to the pathophysiology of adipose tissue inflammation.

\section{Homeostatic Inflammation and Other Metabolic Disorders}

Recent evidence has provided new insight into the interaction between endogenous ligands and pathogen sensors in a variety of chronic inflammatory diseases such as atherosclerosis, diabetes mellitus, malignant cancers, autoimmune diseases, and even neurodegenerative diseases. Similar to the interaction between saturated fatty acids and TLR4, oxidized low-density lipoprotein (LDL), known as a ligand for the scavenger receptor $\mathrm{CD} 36$, is reported to trigger inflammatory signaling through a newly identified heterodimer of TLR4 and TLR6 in macrophages [57] and also to trigger CD36TLR2-dependent apoptosis in macrophages under endoplasmic reticulum stress [58]. On the other hand, Schulthess et al. reported that CXC motif chemokine ligand-10 (CXCL10), when upregulated in diabetic pancreatic islet, is capable of binding to TLR4 in $\beta$ cells in pancreatic islets to induce apoptosis [59].

In Nod-like receptor family, the NACHT, LRR, and PYD domain-containing protein 3 (NLRP3) inflammasome is well characterized. The NLRP3 inflammasome is a cytosolic protein complex consisting of the regulatory subunit NLRP3, the adaptor protein apoptosis-associated speck-like protein containing a caspase-recruitment domain (ASC) and the effector subunit caspase-1. It is activated by pathogen-derived DNA and endogenous DAMPs such as components of necrotic cells and damaged tissues $[60,61]$. Several lines of evidence have suggested that the NLRP3 inflammasome plays an important role in the pathogenesis of obesity-related diseases. NLRP3 deficient mice show improved glucose tolerance and insulin sensitivity [62]. It is also reported that ceramide and islet amyloid polypeptide (IAPP) activate as danger signals for NLRP3 inflammasome in adipose tissue macrophages and pancreatic islets, respectively, which results in insulin resistance [63-65]. In atherogenesis, it is reported that crystalline cholesterol acts as an endogenous danger signal and its deposition in arteries or elsewhere is an early cause rather than a late consequence of inflammation [66]. A better understanding of the molecular basis underlying homeostatic inflammation would allow more efficient multidisciplinary approach to and a better assessment of the metabolic syndrome.

\section{Concluding Remarks}

Obesity may be viewed as a chronic low-grade inflammation as well as a metabolic disease. Although considerable progress has been made in understanding the cellular and molecular events that are involved in acute inflammation caused by infection, there is no clear understanding of their physiological counterpart of the systemic chronic inflammatory state, which could be referred as homeostatic inflammation. The interaction between parenchymal and stromal cells through a number of endogenous ligands and pathogen sensors may contribute to inflammatory responses in obese adipose tissue as well as other metabolic organs. Understanding 
the molecular mechanism underlying adipose tissue remodeling as homeostatic inflammation may lead to novel therapeutic strategies to prevent or treat obesity-related complications.

\section{Abbreviations}

Angptl2: Angiopoietin-like protein 2

ASC: Apoptosis-associated speck-like protein containing a caspase-recruitment domain

ATF3: Activating transcription factor 3

CCR2: Chemokine receptor 2

CXCL10: CXC motif chemokine ligand-10

CXCL14: CXC motif chemokine ligand-14

DAMP: Damage-associated molecular pattern

ECM: $\quad$ Extracellular matrix

FFA: Free fatty acid

IAPP: Islet amyloid polypeptide

IL-4: Interleukin-4

IL-6: Interleukin-6

IL-10: Interleukin-10

IL-13: Interleukin-13

IRF4: Interferon-regulatory factor 4

IRF5: Interferon-regulatory factor 5

Jmjd3: Jumonji domain containing-3

LDL: Low-density lipoprotein

LPS: Lipopolysaccharide

MAPK: Mitogen-activated protein kinase

MCP-1: Monocyte chemoattractant protein-1

NF- $\kappa$ B: $\quad$ Nuclear factor $-\kappa \mathrm{B}$

NLRP3: NACHT, LRR and PYD domain-containing protein 3

PAMP: Pathogen-associated molecular pattern

PPAR $\gamma:$ Peroxisome proliferator-activated receptor $\gamma$

$\operatorname{PPAR} \beta / \delta$ : Peroxisome proliferator-activated receptor $\beta / \delta$

PRR: $\quad$ Pattern-recognition receptor

SVF: $\quad$ Stromal vascular fraction

TLR4: Toll-like receptor 4

TNF $\alpha$ : Tumor necrosis factor- $\alpha$.

\section{Acknowledgments}

Work in the authors' laboratory was supported in part by a Grant-in-Aid for Scientific Research from the Ministry of Education, Culture, Sports, Science, and Technology of Japan, and the Ministry of Health, Labor, and Welfare of Japan.

\section{References}

[1] S. Schenk, M. Saberi, and J. M. Olefsky, "Insulin sensitivity: modulation by nutrients and inflammation," Journal of Clinical Investigation, vol. 118, no. 9, pp. 2992-3002, 2008.

[2] G. S. Hotamisligil, "Inflammation and metabolic disorders," Nature, vol. 444, no. 7121, pp. 860-867, 2006.

[3] A. H. Berg and P. E. Scherer, "Adipose tissue, inflammation, and cardiovascular disease," Circulation Research, vol. 96, no. 9, pp. 939-949, 2005.
[4] V. Z. Rocha and P. Libby, "Obesity, inflammation, and atherosclerosis," Nature Reviews Cardiology, vol. 6, no. 6, pp. 399409, 2009.

[5] Y. Matsuzawa, T. Funahashi, and T. Nakamura, "Molecular mechanism of metabolic syndrome X: contribution of adipocytokines-adipocyte-derived bioactive substances," Annals of the New York Academy of Sciences, vol. 892, pp. 146-154, 1999.

[6] R. Medzhitov, "Origin and physiological roles of inflammation," Nature, vol. 454, no. 7203, pp. 428-435, 2008.

[7] S. Nishimura, I. Manabe, M. Nagasaki et al., "Adipogenesis in obesity requires close interplay between differentiating adipocytes, stromal cells, and blood vessels," Diabetes, vol. 56, no. 6, pp. 1517-1526, 2007.

[8] S. Nishimura, I. Manabe, M. Nagasaki et al., "In vivo imaging in mice reveals local cell dynamics and inflammation in obese adipose tissue," Journal of Clinical Investigation, vol. 118, no. 2, pp. 710-721, 2008.

[9] R. Medzhitov and C. A. Janeway Jr., "Decoding the patterns of self and nonself by the innate immune system," Science, vol. 296, no. 5566, pp. 298-300, 2002.

[10] X. Zhang and D. M. Mosser, "Macrophage activation by endogenous danger signals," Journal of Pathology, vol. 214, no. 2, pp. 161-178, 2008.

[11] T. Suganami and Y. Ogawa, "Adipose tissue macrophages: their role in adipose tissue remodeling," Journal of Leukocyte Biology, vol. 88, no. 1, pp. 33-39, 2010.

[12] F. Wasserman, Handbook of Physiology, American Physiology Society, Washington, DC, USA, 1965.

[13] G. H. Gibbons and V. J. Dzau, "The emerging concept of vascular remodeling," The New England Journal of Medicine, vol. 330, no. 20, pp. 1431-1438, 1994.

[14] S. P. Weisberg, D. McCann, M. Desai, M. Rosenbaum, R. L.Leibel, and A. W. Ferrante Jr., "Obesity is associated with macrophage accumulation in adipose tissue," Journal of Clinical Investigation, vol. 112, no. 12, pp. 1796-1808, 2003.

[15] H. Xu, G. T. Barnes, Q. Yang et al., "Chronic inflammation in fat plays a crucial role in the development of obesity-related insulin resistance," Journal of Clinical Investigation, vol. 112, no. 12, pp. 1821-1830, 2003.

[16] S. P. Weisberg, D. Hunter, R. Huber et al., "CCR2 modulates inflammatory and metabolic effects of high-fat feeding," Journal of Clinical Investigation, vol. 116, no. 1, pp. 115-124, 2006.

[17] H. Kanda, S. Tateya, Y. Tamori et al., "MCP-1 contributes to macrophage infiltration into adipose tissue, insulin resistance, and hepatic steatosis in obesity," Journal of Clinical Investigation, vol. 116, no. 6, pp. 1494-1505, 2006.

[18] N. Kamei, K. Tobe, R. Suzuki et al., "Overexpression of monocyte chemoattractant protein-1 in adipose tissues causes macrophage recruitment and insulin resistance," Journal of Biological Chemistry, vol. 281, no. 36, pp. 26602-26614, 2006.

[19] A. Ito, T. Suganami, A. Yamauchi et al., "Role of CC chemokine receptor 2 in bone marrow cells in the recruitment of macrophages into obese adipose tissue," Journal of Biological Chemistry, vol. 283, no. 51, pp. 35715-35723, 2008.

[20] T. Nomiyama, D. Perez-Tilve, D. Ogawa et al., "Osteopontin mediates obesity-induced adipose tissue macrophage infiltration and insulin resistance in mice," Journal of Clinical Investigation, vol. 117, no. 10, pp. 2877-2888, 2007.

[21] N. Nara, Y. Nakayama, S. Okamoto et al., "Disruption of CXC motif chemokine ligand-14 in mice ameliorates obesityinduced insulin resistance," Journal of Biological Chemistry, vol. 282, no. 42, pp. 30794-30803, 2007. 
[22] M. Tabata, T. Kadomatsu, S. Fukuhara et al., "Angiopoietinlike protein 2 promotes chronic adipose tissue inflammation and obesity-related systemic insulin resistance," Cell Metabolism, vol. 10, no. 3, pp. 178-188, 2009.

[23] T. Suganami, J. Nishida, and Y. Ogawa, "A paracrine loop between adipocytes and macrophages aggravates inflammatory changes: role of free fatty acids and tumor necrosis factor $\alpha$," Arteriosclerosis, Thrombosis, and Vascular Biology, vol. 25, no. 10, pp. 2062-2068, 2005.

[24] T. Suganami, K. Tanimoto-Koyama, J. Nishida et al., "Role of the toll-like receptor $4 / \mathrm{NF}-\kappa \mathrm{B}$ pathway in saturated fatty acidinduced inflammatory changes in the interaction between adipocytes and macrophages," Arteriosclerosis, Thrombosis, and Vascular Biology, vol. 27, no. 1, pp. 84-91, 2007.

[25] T. Yamauchi, J. Kamon, H. Waki et al., "The fat-derived hormone adiponectin reverses insulin resistance associated with both lipoatrophy and obesity," Nature Medicine, vol. 7, no. 8, pp. 941-946, 2001.

[26] N. Maeda, I. Shimomura, K. Kishida et al., "Diet-induced insulin resistance in mice lacking adiponectin/ACRP30," Nature Medicine, vol. 8, no. 7, pp. 731-737, 2002.

[27] S. Tateya, Y. Tamori, T. Kawaguchi, H. Kanda, and M. Kasuga, "An increase in the circulating concentration of monocyte chemoattractant protein-1 elicits systemic insulin resistance irrespective of adipose tissue inflammation in mice," Endocrinology, vol. 151, no. 3, pp. 971-979, 2010.

[28] C. N. Lumeng, J. L. Bodzin, and A. R. Saltiel, "Obesity induces a phenotypic switch in adipose tissue macrophage polarization," Journal of Clinical Investigation, vol. 117, no. 1, pp. 175-184, 2007.

[29] J. M. Olefsky and C. K. Glass, "Macrophages, inflammation, and insulin resistance," Annual Review of Physiology, vol. 72, pp. 219-246, 2010.

[30] T. Suganami, X. Yuan, Y. Shimoda et al., "Activating transcription factor 3 constitutes a negative feedback mechanism that attenuates saturated fatty acid/toll-like receptor 4 signaling and macrophage activation in obese adipose tissue," Circulation Research, vol. 105, no. 1, pp. 25-32, 2009.

[31] J. I. Odegaard, R. R. Ricardo-Gonzalez, M. H. Goforth et al., "Macrophage-specific PPAR $\gamma$ controls alternative activation and improves insulin resistance," Nature, vol. 447, no. 7148, pp. 1116-1120, 2007.

[32] J. I. Odegaard, R. R. Ricardo-Gonzalez, A. Red Eagle et al., "Alternative M2 activation of kupffer cells by PPAR $\delta$ ameliorates obesity-induced insulin resistance," Cell Metabolism, vol. 7, no. 6, pp. 496-507, 2008.

[33] K. Kang, S. M. Reilly, V. Karabacak et al., "Adipocyte-derived Th2 cytokines and myeloid $\operatorname{PPAR} \delta$ regulate macrophage polarization and insulin sensitivity," Cell Metabolism, vol. 7, no. 6, pp. 485-495, 2008.

[34] D. Vats, L. Mukundan, J. I. Odegaard et al., "Oxidative metabolism and PGC- $\beta$ attenuate macrophage-mediated inflammation," Cell Metabolism, vol. 4, no. 1, pp. 13-24, 2006.

[35] S. Fujisaka, I. Usui, A. Bukhari et al., "Regulatory mechanisms for adipose tissue M1 and M2 macrophages in diet-induced obese mice," Diabetes, vol. 58, no. 11, pp. 2574-2582, 2009.

[36] N. Satoh, A. Shimatsu, A. Himeno et al., "Unbalanced M1/M2 phenotype of peripheral blood monocytes in obese diabetic patients: effect of pioglitazone," Diabetes Care, vol. 33, no. 1, p. e7, 2010.

[37] M. A. Bouhlel, B. Derudas, E. Rigamonti et al., "PPAR $\gamma$ activation primes human monocytes into alternative M2 macrophages with anti-inflammatory properties," Cell Metabolism, vol. 6, no. 2, pp. 137-143, 2007.
[38] T. Satoh, O. Takeuchi, A. Vandenbon et al., "The Jmjd3-Irf4 axis regulates M2 macrophage polarization and host responses against helminth infection," Nature Immunology, vol. 11, no. 10, pp. 936-944, 2010.

[39] T. Krausgruber, K. Blazek, T. Smallie et al., "IRF5 promotes inflammatory macrophage polarization and $\mathrm{T}(\mathrm{H}) 1-\mathrm{T}(\mathrm{H}) 17$ responses," Nature Immunology, vol. 12, no. 3, pp. 231-238, 2011.

[40] C. Henegar, J. Tordjman, V. Achard et al., "Adipose tissue transcriptomic signature highlights the pathological relevance of extracellular matrix in human obesity," Genome Biology, vol. 9, no. 1, Article ID R14, 2008.

[41] D. M. Mutch, J. Tordjman, V. Pelloux et al., "Needle and surgical biopsy techniques differentially affect adipose tissue gene expression profiles," The American Journal of Clinical Nutrition, vol. 89, no. 1, pp. 51-57, 2009.

[42] J. Liu, A. Divoux, J. Sun et al., "Genetic deficiency and pharmacological stabilization of mast cells reduce diet-induced obesity and diabetes in mice," Nature Medicine, vol. 15, no. 8, pp. 940-945, 2009.

[43] T. Khan, E. S. Muise, P. Iyengar et al., "Metabolic dysregulation and adipose tissue fibrosis: role of collagen VI," Molecular and Cellular Biology, vol. 29, no. 6, pp. 1575-1591, 2009.

[44] A. Divoux, J. Tordjman, D. Lacasa et al., "Fibrosis in human adipose tissue: composition, distribution, and link with lipid metabolism and fat mass loss," Diabetes, vol. 59, no. 11, pp. 2817-2825, 2010.

[45] M. Y. Wang, P. Grayburn, S. Chen, M. Ravazzola, L. Orci, and R. H. Unger, "Adipogenic capacity and the susceptibility to type 2 diabetes and metabolic syndrome," Proceedings of the National Academy of Sciences of the United States of America, vol. 105, no. 16, pp. 6139-6144, 2008.

[46] K. J. Strissel, Z. Stancheva, H. Miyoshi et al., "Adipocyte death, adipose tissue remodeling, and obesity complications," Diabetes, vol. 56, no. 12, pp. 2910-2918, 2007.

[47] C. Duval, U. Thissen, S. Keshtkar et al., "Adipose tissue dysfunction signals progression of hepatic steatosis towards nonalcoholic steatohepatitis in C57Bl/6 mice," Diabetes, vol. 59, no. 12, pp. 3181-3191, 2010.

[48] L. Shi, R. Kishore, M. R. McMullen, and L. E. Nagy, "Lipopolysaccharide stimulation of ERK1/2 increases TNF- $\alpha$ production via Egr-1," American Journal of Physiology-Cell Physiology, vol. 282, no. 6, pp. C1205-C1211, 2002.

[49] M. Poggi, D. Bastelica, P. Gual et al., "C $3 \mathrm{H} / \mathrm{HeJ}$ mice carrying a toll-like receptor 4 mutation are protected against the development of insulin resistance in white adipose tissue in response to a high-fat diet," Diabetologia, vol. 50, no. 6, pp. 1267-1276, 2007.

[50] D. M. Tsukumo, M. A. Carvalho-Filho, J. B. Carvalheira et al., "Loss-of-function mutation in toll-like receptor 4 prevents diet-induced obesity and insulin resistance," Diabetes, vol. 56, no. 8, pp. 1986-1998, 2007.

[51] J. Y. Lee, K. H. Sohn, S. H. Rhee, and D. Hwang, "Saturated fatty acids, but not unsaturated fatty acids, induce the expression of cyclooxygenase-2 mediated through toll-like receptor 4," Journal of Biological Chemistry, vol. 276, no. 20, pp. 16683-16689, 2001.

[52] M. Itoh, T. Suganami, N. Satoh et al., "Increased adiponectin secretion by highly purified eicosapentaenoic acid in rodent models of obesity and human obese subjects," Arteriosclerosis, Thrombosis, and Vascular Biology, vol. 27, no. 9, pp. 19181925, 2007.

[53] A. Kosteli, E. Sugaru, G. Haemmerle et al., "Weight loss and lipolysis promote a dynamic immune response in murine 
adipose tissue," Journal of Clinical Investigation, vol. 120, no. 10, pp. 3466-3479, 2010.

[54] M. Ichioka, T. Suganami, N. Tsuda et al., "Increased expression of macrophage-inducible C-type lectin in adipose tissue of obese mice and humans," Diabetes, vol. 60, no. 3, pp. 819-826, 2011.

[55] S. Yamasaki, E. Ishikawa, M. Sakuma, H. Hara, K. Ogata, and T. Saito, "Mincle is an ITAM-coupled activating receptor that senses damaged cells," Nature Immunology, vol. 9, no. 10, pp. 1179-1188, 2008.

[56] S. Cinti, G. Mitchell, G. Barbatelli et al., "Adipocyte death defines macrophage localization and function in adipose tissue of obese mice and humans," Journal of Lipid Research, vol. 46, no. 11, pp. 2347-2355, 2005.

[57] C. R. Stewart, L. M. Stuart, K. Wilkinson et al., "CD36 ligands promote sterile inflammation through assembly of a toll-like receptor 4 and 6 heterodimer," Nature Immunology, vol. 11, no. 2, pp. 155-161, 2010.

[58] T. A. Seimon, M. J. Nadolski, X. Liao et al., "Atherogenic lipids and lipoproteins trigger CD36-TLR2-dependent apoptosis in macrophages undergoing endoplasmic reticulum stress," Cell Metabolism, vol. 12, no. 5, pp. 467-482, 2010.

[59] F. T. Schulthess, F. Paroni, N. S. Sauter et al., "CXCL10 impairs $\beta$ cell function and viability in diabetes through TLR4 signaling," Cell Metabolism, vol. 9, no. 2, pp. 125-139, 2009.

[60] K. Schroder and J. Tschopp, "The Inflammasomes," Cell, vol. 140, no. 6, pp. 821-832, 2010.

[61] K. L. Rock, E. Latz, F. Ontiveros, and H. Kono, "The sterile inflammatory response," Annual Review of Immunology, vol. 28, pp. 321-342, 2010.

[62] R. Zhou, A. Tardivel, B. Thorens, I. Choi, and J. Tschopp, "Thioredoxin-interacting protein links oxidative stress to inflammasome activation," Nature Immunology, vol. 11, no. 2, pp. 136-140, 2010.

[63] R. Stienstra, L. A. Joosten, T. Koenen et al., "The inflammasome-mediated caspase-1 activation controls adipocyte differentiation and insulin sensitivity," Cell Metabolism, vol. 12, no. 6, pp. 593-605, 2010.

[64] B. Vandanmagsar, Y.-H. Youm, A. Ravussin et al., "The NLRP3 inflammasome instigates obesity-induced inflammation and insulin resistance," Nature Medicine, vol. 17, no. 2, pp. 179189, 2011.

[65] S. L. Masters, A. Dunne, and S. L. Subramanian, "Activation of the NLRP3 inflammasome by islet amyloid polypeptide provides a mechanism for enhanced IL- $1 \beta$ in type 2 diabetes," Nature Immunology, vol. 11, no. 10, pp. 897-904, 2010.

[66] P. Duewell, H. Kono, K. J. Rayner et al., "NLRP3 inflammasomes are required for atherogenesis and activated by cholesterol crystals," Nature, vol. 464, no. 7293, pp. 1357-1361, 2010. 


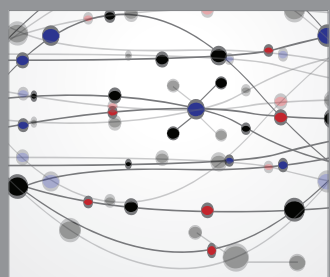

The Scientific World Journal
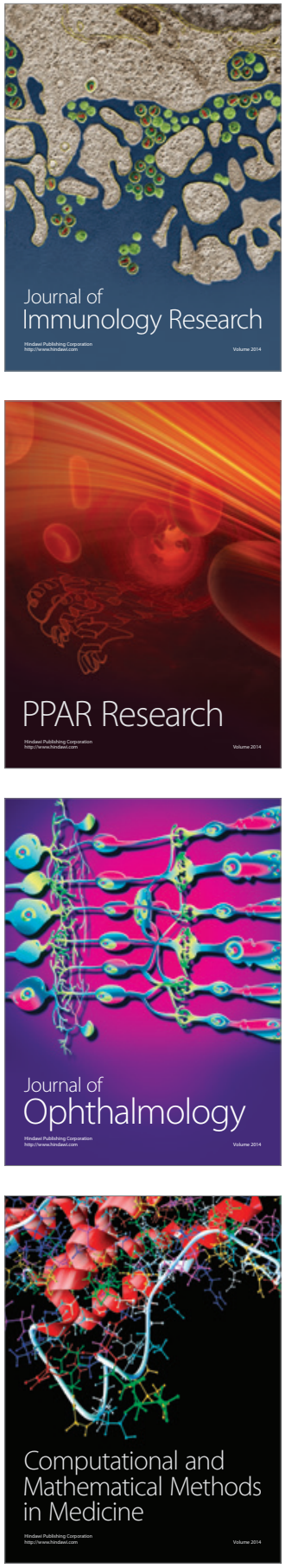

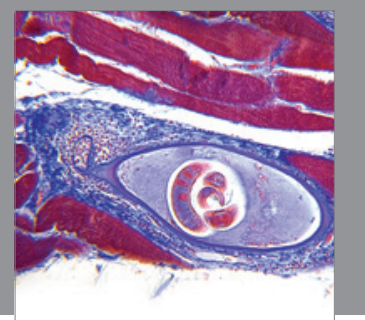

Gastroenterology

Research and Practice
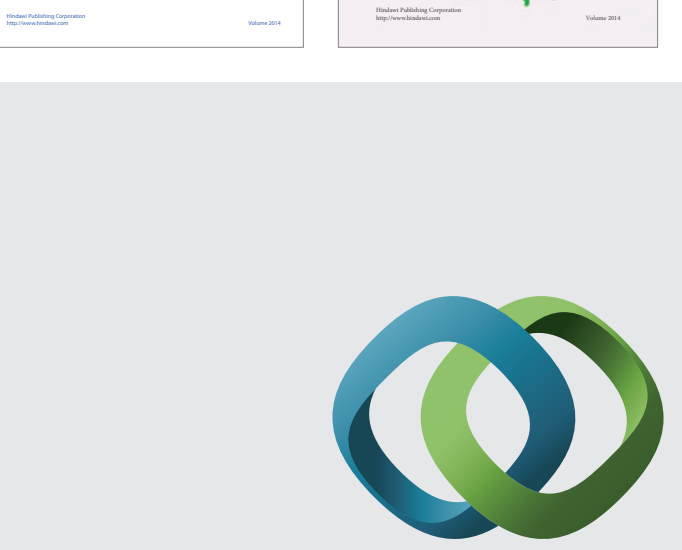

\section{Hindawi}

Submit your manuscripts at

http://www.hindawi.com
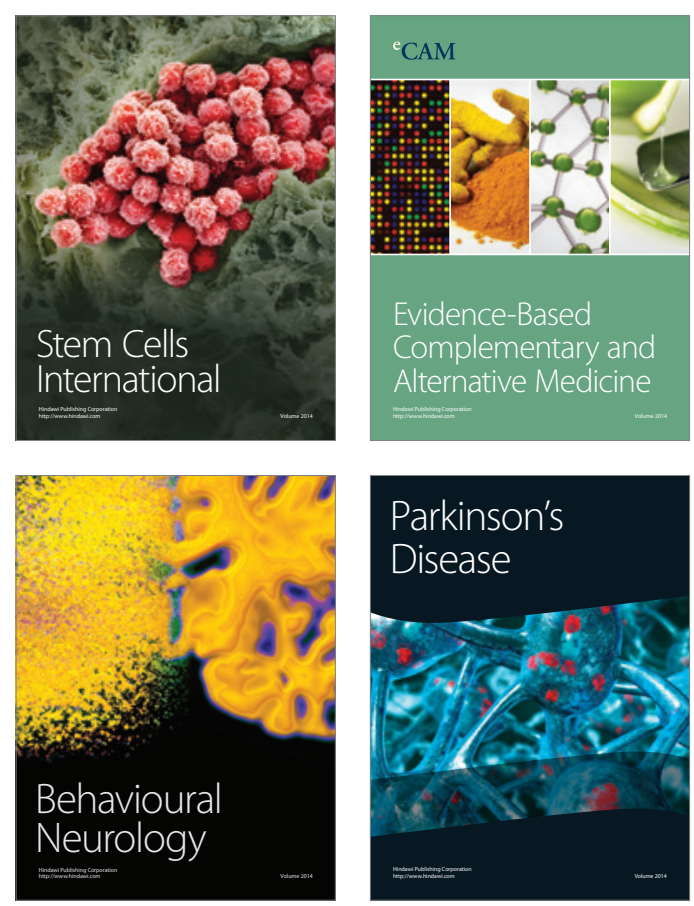

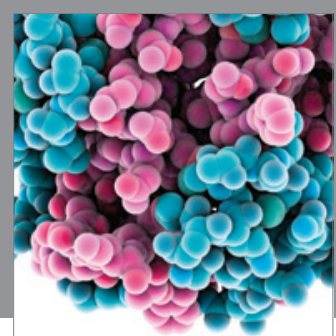

Journal of
Diabetes Research

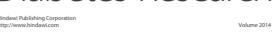

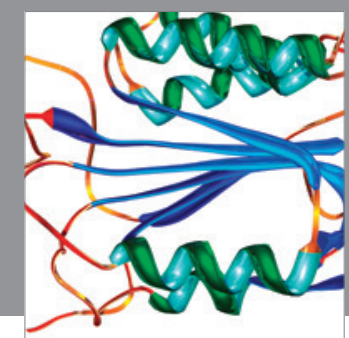

Disease Markers
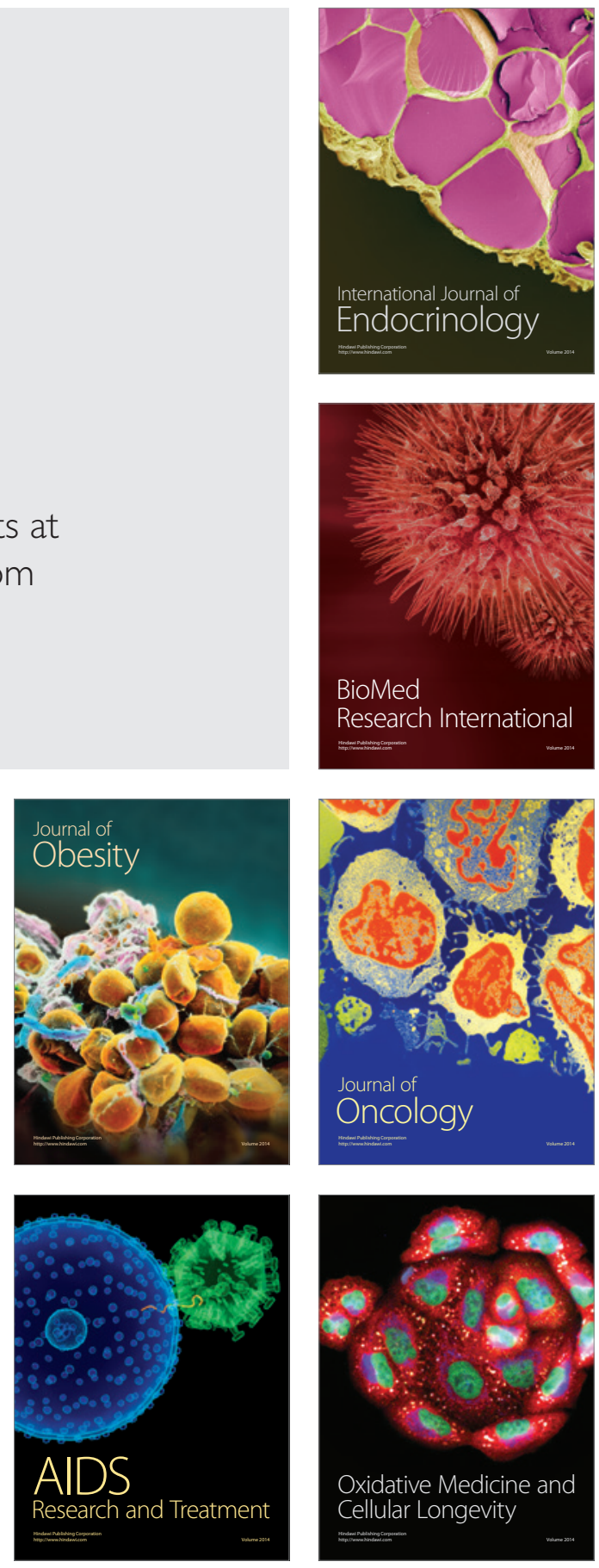